\title{
Ventricular stimulation, but from where?
}

\author{
Raghav Bansal ${ }^{1}$, Ramalingam Vadivelu ${ }^{1}$, Eduardo Sternick $^{2}$, and Yash Lokhandawala ${ }^{1}$ \\ ${ }^{1}$ Holy Family Hospital \\ ${ }^{2}$ Biocor Inst
}

October 14, 2020

\section{EP Rounds}

Ventricular stimulation, but from where?

Raghav Bansal, MD, DM ${ }^{1}$; Ramalingam Vadivelu, MD, DM ${ }^{1}$; Eduardo Sternick, MD, PhD $^{2}$; Yash Lokhandwala, MD, DM ${ }^{1}$

${ }^{1}$ Department of Cardiology, Holy Family Hospital, Hill Road, Bandra West, Mumbai - 400050, India

${ }^{2}$ Department of Arrhyhthmia and Electrophysiology, Biocor Institute, NovaLima, Brazil

Corresponding author: Ramalingam Vadivelu, MD, DM Department of Cardiology, Holy Family Hospital,

Hill Road, Bandra West, Mumbai - 400050, India

Contact: $+91-8156843960$

e-mail: vvelu00@gmail.com

Word Count (excluding references and figure legends): 706

There are no conflicts of interest to state

\section{Funding: None}

\section{Case Presentation}

A 16-year-old boy was referred for an electrophysiological study in view of rapid episodic palpitations with documented regular narrow complex tachycardia. He had no manifest pre-excitation. Clinical tachycardia with cycle length of $360 \mathrm{~ms}$, was easily inducible by premature atrial complexes. A diagnosis of a concealed left lateral accessory pathway was made with an eccentric atrial activation sequence both during tachycardia and right ventricular (RV) pacing (Figure 1, left-hand panel). The pathway was mapped at the left posterior mitral vestibule during RV pacing, performed through the distal tip of the His bundle catheter (Figure 1, right panel). Intracardiac electrograms at the start of radiofrequency $(\mathrm{RF})$ energy are depicted in the left hand panel of Figure 2. As the energy continues, shown in the right-hand panel of Figure 2, there is an alternation of QRS complex width and morphology. How is this explained?

\section{Discussion}

The right panel in Figure 1 depicts the site of ablation of a concealed left free-wall accessory pathway (red arrow) by a retrograde transaortic approach. Ablation during ventricular pacing is preferable compared to during sustained tachycardia in view of the advantage of catheter stability, especially after elimination of the pathway during ablation. Notably, pacing was performed just below the RV basal outflow tract with the His 
bundle catheter pushed distally (white arrowhead, right-hand panels of Figure 1). The ablation signal in the left panel of Figure 2 marks the 'A' with an arrow, the small potential just after the ventricular electrogram in the RF distal channel. The early atrial activation along with the absence of isoelectric interval between $\mathrm{V}$ and A suggest that it is likely to be a successful ablation site.

With initiation of RF energy, there was an increase in the ventriculo-atrial interval in the distal coronary sinus (CS12) and RF distal channels with a change in atrial activation sequence (Figure 2, left panel). During continuation of ablation, the alternate wide and narrow QRS beats have the same retrograde concentric atrial activation pattern with a constant VA interval, as depicted in Figure 3. The narrower QRS complexes are associated with a reversal of the ventricular activation sequence in the coronary sinus channels. This may occur due to one of the following mechanisms: i) alternate His bundle capture and non-capture; ii) capture of the antero-basal left ventricle along with the RV on alternate beats; iii) RF catheter induced premature ventricular complexes (PVCs), causing fusion and narrowing of the alternate beats. His bundle capture is unlikely in this case as the catheter was placed deeper in the RV base and there was no His signal in the His distal channel at baseline. Capture of the antero-basal left ventricle on alternate beats resulting in narrow QRS is a possibility; however, the ventricular electrogram in the RF catheter would not be coincident with the pacing stimulus, seeing the large distance between the RF catheter and the His catheter (Figure 1, righthand panel). Catheter induced PVCs have been reported to occur in a bigeminal pattern ${ }^{1}$ due to cardiac motion during tachycardia or ventricular pacing and then fuse with the paced beat, resulting in a narrower QRS complex. The earliest ventricular activation in the RF distal channel followed by ventricular activation wave front from distal to proximal in the CS suggests catheter induced PVCs as a putative mechanism in this case.

The change in the atrial activation pattern from eccentric to concentric is consistent with elimination of accessory pathway conduction. However, residual accessory pathway conduction may be missed by RV pacing. Interestingly, the phenomenon of alternating narrow and wide QRS complexes allows insight into the assessment of the success of RF ablation in this case. The atrial activation pattern and the stimulus to atrial activation interval remains exactly the same despite early left ventricular free wall capture. Note in the RF distal signal in Figure 3, the presence of four different potentials: the first immediately following the pacing spike is the local ventricular potential $(\mathrm{V})$, follow by a likely artefact, followed by an atrial potential (A) and another potential $(*)$. This additional electrogram marked by an asterisk is possibly a split atrial electrogram.

Acknowledgement: The authors wish to acknowledge the help in the interpretation provided by Prof Hein JJ Wellens prior to his unfortunate demise.

\section{References}

Bhargava K, Jindal R, Kler TS. Narrow QRS tachycardia with alternate wide QRS beats: What is the mechanism? Indian Pacing Electrophysiol J . 2008;8(3):234-237.

\section{Figure Legends}

Figure 1. Left hand panel. Mapping during right ventricular pacing revealing left lateral accessory pathway with eccentric atrial activation noted in the CS channels. Right hand panel.Fluoroscopic view demonstrating position of catheters in RAO $30^{\circ}$ view (upper figure) and LAO $40^{\circ}$ view (lower figure). The red arrow marks the tip of radiofrequency ablation catheter and the white arrow marks the position of the pacing catheter inside the right ventricle.

Figure 2. Left hand panel. Intracardiac electrograms at the start of radiofrequency energy application. Note that the atrial activation pattern changes on the $2^{\text {nd }}$ beat after initiation of ablation, suggesting a successful site. Also note, the last QRS complex is narrower. Right hand panel. Note the alternating wide and narrow QRS morphology.

Figure 3. Intracardiac electrograms during radiofrequency energy application, the same as the right-hand panel of Figure 2. Note that ventricular activation in the CS channels change along with alternating wide 
and narrow QRS morphology. Also note that after the narrow QRS beat multiple electrograms follow the ventricular electrogram in the RF distal channel. The first one is likely to be an artefact, the second and third ones being split atrial potentials.
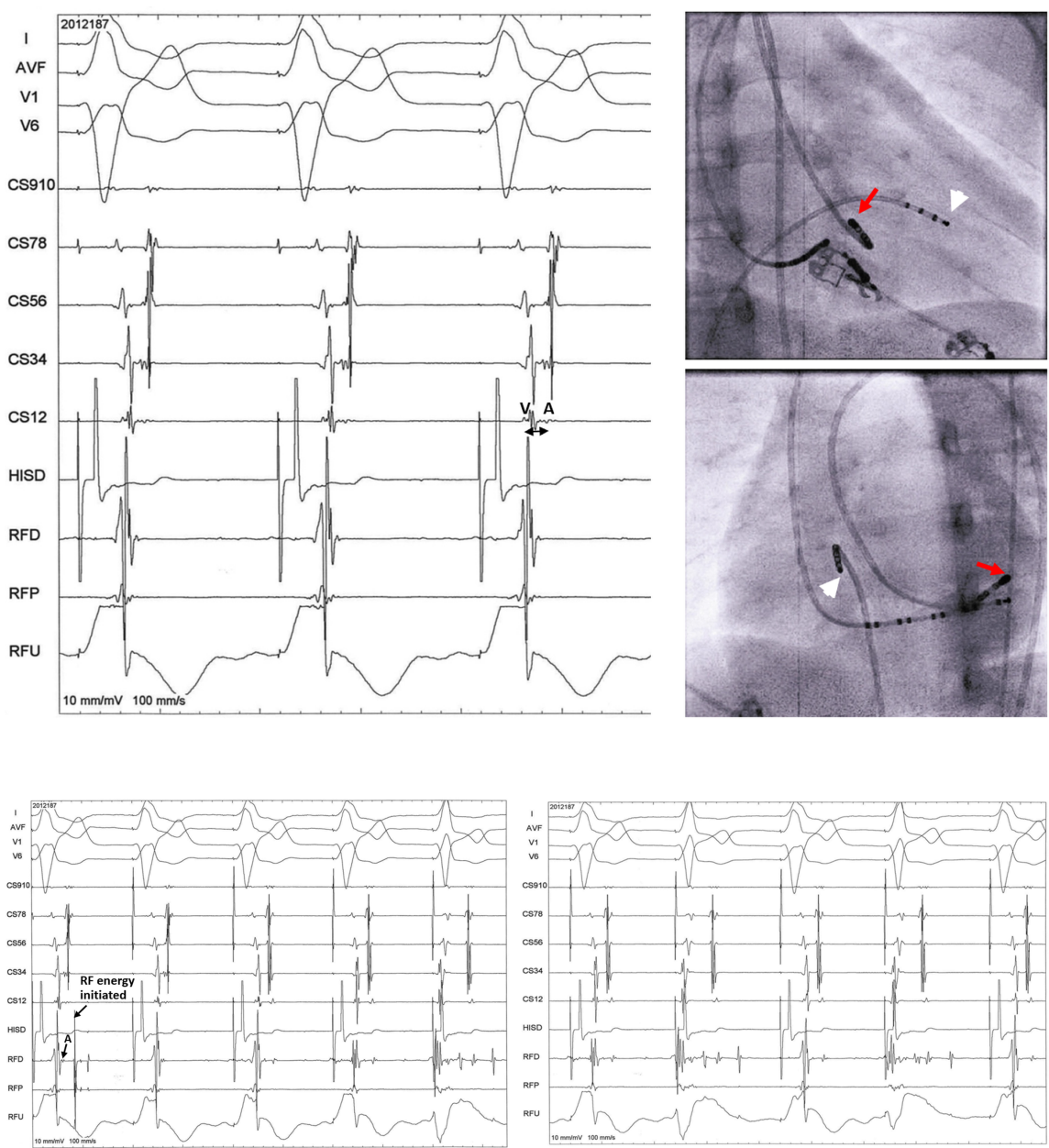


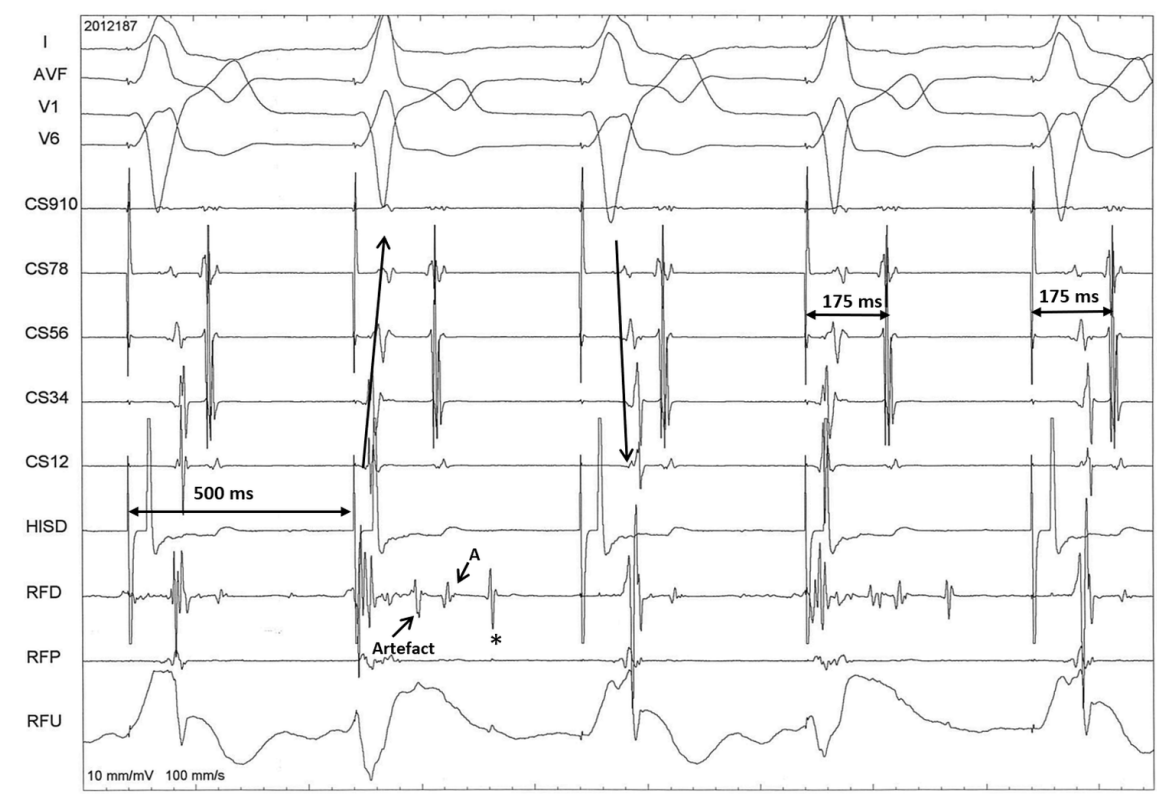

